\title{
TAXI: A VERSATILE PROCESSING CHAIN FOR EXPERIMENTAL TANDEM-X PRODUCT EVALUATION
}

\author{
Pau Prats, Marc Rodriguez-Cassola, Luca Marotti, Matteo Naninni, Steffen Wollstadt, Daniel Schulze, \\ Núria Tous-Ramon, Marwan Younis, Gerhard Krieger, and Andreas Reigber
}

\author{
Microwaves and Radar Institute \\ German Aerospace Center
}

\begin{abstract}
TanDEM-X is a high-resolution interferometric radar mission with the main goal of providing a global digital elevation model (DEM) of the Earth surface by means of single-pass $\mathrm{X}$-band SAR interferometry. It is, moreover, the first genuinely bistatic spaceborne SAR mission, and, independently of its usual quasi-monostatic configuration, includes many of the peculiarities of bistatic SAR. An experimental, versatile, and flexible interferometric chain has been developed at DLR Microwaves and Radar Institute for the scientific exploitation of TanDEM-X data acquired in non-standard configurations. The paper describes the structure of the processing chain and focusses on some essential aspects of its bistatic part. Some experimental results performed with TerraSAR-X demonstrate the flexibility of the implemented processor.
\end{abstract}

Index Terms - TanDEM-X, bistatic, interferometry

\section{INTRODUCTION}

TanDEM-X is the first bistatic SAR mission in space [1]. The main objective of the mission is to act as a single-pass interferometric system capable of providing very high resolution $3 \mathrm{D}$ information. As it is widely recognised, bistatic SARs offer increased performance at an increased operational cost. The uniqueness of the mission, coupled with the flexibility of the TerraSAR-X (TSX) and TanDEM-X (TDX) satellites, allows one to develop a large amount of challenging experiments to take advantage of the potential of the system. For the evaluation of these experiments, a flexible interferometric chain is being developed at the Microwaves and Radar Institute of DLR (DLR-HR) to exploit the novel scientific data that will be gathered during the mission. We have named this processor TAXI (experimental TanDEM-X interferometric processor).

The paper addresses the particular characteristics of the processing chain, especially focussed on its bistatic part. First, a block diagram of the processing chains is presented, followed by a discussion of the core of the bistatic processing part. The selected synchronisation approach, imaging modes

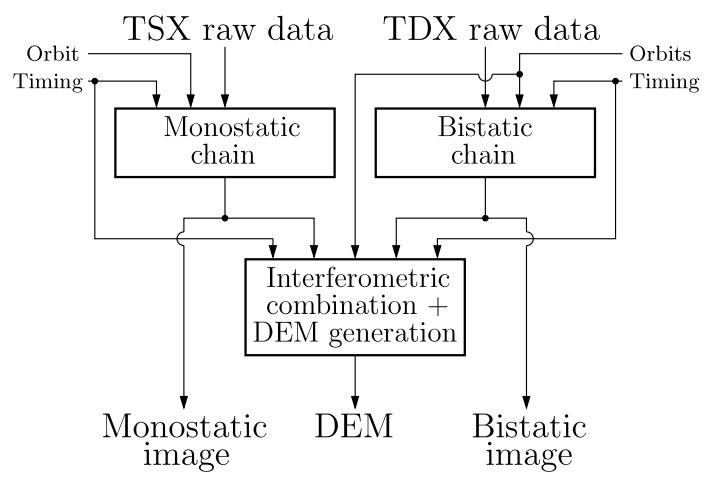

Fig. 1. Block diagram of TAXI.

and processing kernels are also presented, including a performance analysis as a function of the key parameters of the system. Section 4 will present some experimental results performed by TSX, with the aim of exemplifying the flexibility of TAXI.

\section{TAXI: EXPERIMENTAL TANDEM-X INTERFEROMETRIC PROCESSOR}

The TAXI processor for the evaluation of experimental data products, which is being developed at DLR-HR, is a versatile SAR processing chain mainly composed of three main branches: a) a high-precision spaceborne monostatic part for the processing of TSX data, b) a high precision spaceborne bistatic part for the processing of TanDEM-X data, and c) an interferometric part for the combination of a stack of images, either single pass or repeat pass, including the steps of DEM generation and geocoding.

Fig. 1 shows the block diagram of TAXI with its three main blocks. The standard imaging modes of TSX are stripmap, sliding spotlight, and ScanSAR, while TOPS is operated by DLR Microwaves and Radar Institute (DLR-HR) as an experimental mode [2]. Due to the usually quasimonostatic configurations of TanDEM-X, analogous imaging modes extended to the bistatic case can be thought of. The focussing kernels of the monostatic part are based on the 


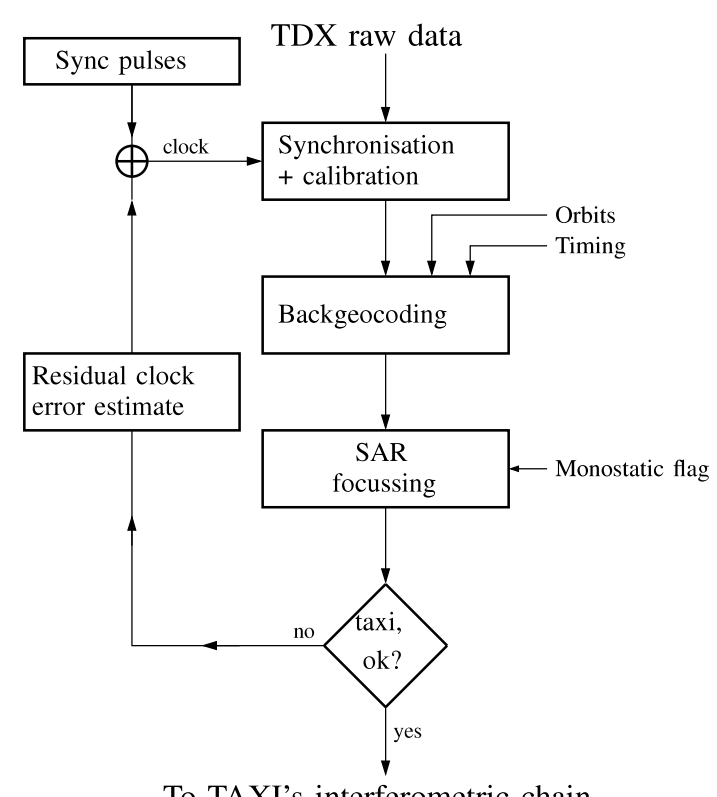

To TAXI's interferometric chain

Fig. 2. Block diagram of the bistatic part of the TanDEM-X interferometric processor.

chirp scaling approach. The so-called extended chirp scaling (ECS) [3] is used for the stripmap configurations, whereas the other monostatic modes, which demand a special azimuth focussing approach, further use the common kernel of the baseband azimuth scaling (BAS) [2]. The interferometric part is composed of a geometrical coregistration (using an external DEM and the orbits), interferogram computation, phase unwrapping and DEM generation and geocoding.

\section{TAXI BISTATIC PROCESSING CHAIN}

TAXI's bistatic chain is a general azimuth-invariant bistatic SAR processor. Its block diagram is shown in Fig. 2. The basic structure consists of a synchronisation/calibration part and a focussing part. A feedback loop to estimate the residual synchronisation information has been included. This strategy is expected to be very useful in the experimental cases where a poor signal-to-noise ratio (SNR) of TanDEM-X synchronisation pulses might degrade the performance of the synchronisation link.

\subsection{Bistatic data synchronisation}

TanDEM-X is a cooperative system. During the acqusitions, the TSX and TDX satellites exchange transmitting pulses along their direct path for synchronisation purposes. An evaluation of these pulses provides information on the clock phase error, namely the frequency offset and the phase noise. This information is used for the synchronisation of the bistatic data, a crucial step if low-error interferograms are desired
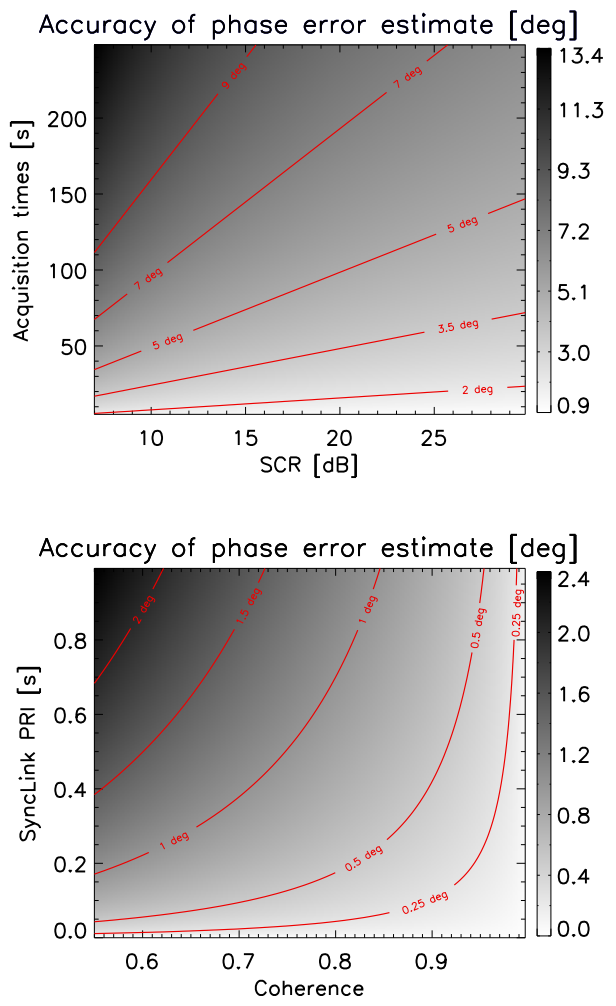

Fig. 3. (top) Accuracy of the phase error estimation as a function of the SCR and the duration of the data take. Noncoherent image combination. (bottom) Accuracy of the phase error estimation as a function of the SyncLink pulse repetition interval (PRI) and scene coherence.

[1]. As expected, the higher the SNR of the synchronisation pulses (closer baselines, low antenna patterns attenuation) and the lower their pulse repetition interval, the lower the error in the estimation of the clock phase error. Due to the particularities of the large baseline configurations, a significant decrease in the SNR of the synchronisation pulses may occur and therefore a further automatic algorithm is foreseen. The automatic synchronisation is based on a direct comparison of the error-free monostatic image and the bistatic one. By measuring the deformation of this bistatic image, an estimate of the residual clock phase error can be obtained. Fig. 3(top) shows the expected accuracy of the automatic synchronisation as a function of the duration of the acquisition and of the SCR of the image. The plot is optimistic, since it is assumed that non-coherent correlation is assumed along the swath for all azimuth blocks. If only one fourth of the range bins could be averaged the accuracy of the measure would worsen by a factor two.

In fact, this automatic synchronisation can be also used in the case of smaller baselines where synchronisation pulses and coherence between the bistatic and monostatic images might be available to reduce the PRF of the synchronisation 


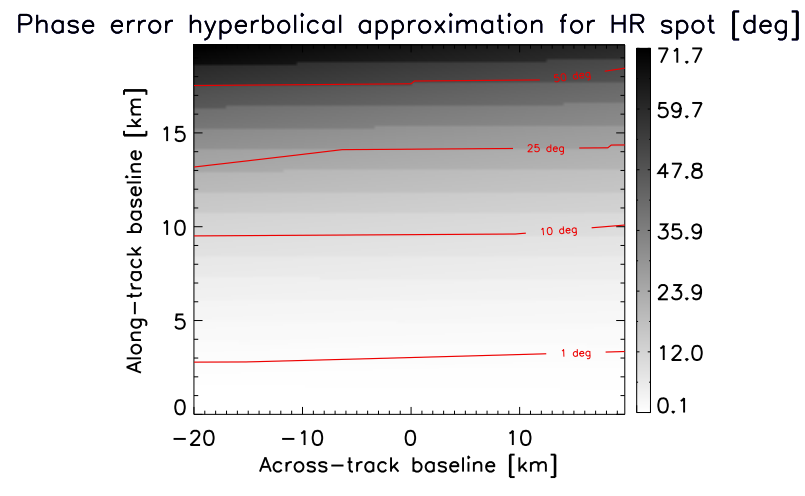

Fig. 4. Maximum phase error at the edge of the synthetic aperture by using the hyperbolical monostatic approximation for a high-resolution spotlight case computed for near range.

link. Fig. 3(bottom) shows the accuracy of the automatic synchronisation as a function of the synchronisation link PRI and of the scene coherence. For an accuracy of the clock phase error estimate better than $1 \mathrm{deg}$, the synchronisation link PRF can be set to $5 \mathrm{~Hz}$ for practical purposes.

\subsection{Bistatic SAR focussing}

TSX and TDX satellites fly in a quasi-azimuth-invariant configuration. Therefore, a Doppler-domain focussing approach is selected. Among the Doppler-domain options for the processing of spaceborne SAR data, a dual-solution approach is used for the bistatic focussing kernels. One option is to tune the monostatic processing functions so that they are matched to the bistatic configuration and then use the regular monostatic kernels, i.e., still using the hyperbolic approximation with an effective velocity [4]. Another one is to generate actual bistatic processing functions using a numerical approach [5]. Fig. 4 shows the maximum phase error at the edge of the synthetic aperture for a high-resolution spotlight acquisition for different combinations of across-track and alongtrack baselines. Whereas the impact of the across-track baseline is mild, an edge error better than $1 \mathrm{deg}$ is expected for the operational configurations of the mission, the along-track baseline has a higher impact on the validity of the approximation.

The imaged scene, including topography, is backgeocoded in the bistatic radar coordinates, a step which is required for focussing and for the geometrical coregistration of the images during interferometric processing. Note that the TanDEM-X baseline is not constant during the acquisition, i.e., TSX and TDX fly in a bistatic azimuth-variant configuration. However, in practical terms, the change of the baseline within one data take is very slow, so we can assume the configuration to be invariant for a certain scene size. If the along-track size of the data take exceeds this invariant scene size, azimuth block processing is required. TAXI's bistatic chain includes

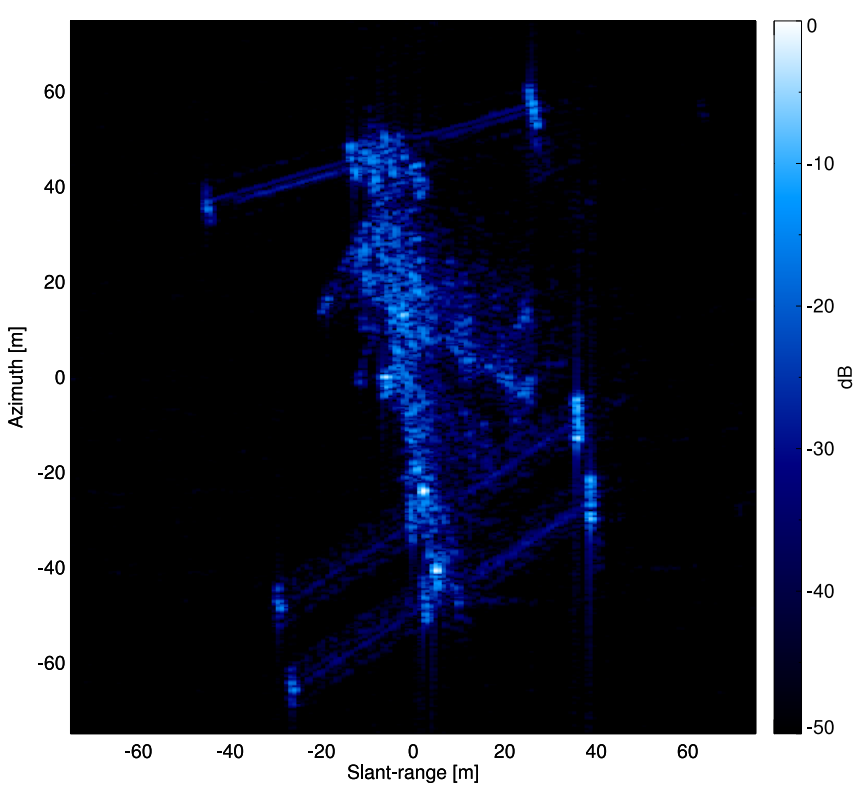

Fig. 5. The ISS as viewd by TSX.

an azimuth-invariance check module to dynamically adapt the block scene size to that of the invariance region of the configuration.

\section{EXPERIMENTAL RESULTS}

This section shows a few experimental results acquired by TSX and processed by TAXI, namely, a data take of the International Space Station (ISS), a differential interferogram acquired with the so-called TOPS mode, and an example of an extended high-resolution mode. It is worth mentioning the flexibility of TSX to accept such complex commandings, as well as the effort in the commanding of the satellite, which due to the non-operational nature of the experiments is everything but trivial.

\subsection{Imaging of the ISS with TerraSAR-X}

On March 13th, 2008, the ISS crossed the field of view of TSX, allowing a unique opportunity to perfom a very special data take. Both the commanding and the processing were challenging, the former due to the necessary monitoring of the ISS for an accurate timing of the data take, and the latter due to the non-hyperbolic phase history of the ISS as seen by TSX, requiring of non-operational solutions to perform the focussing. Both time-domain and frequency-domain approaches were successfully implemented and tested within TAXI. Fig. 5 shows the final result. 


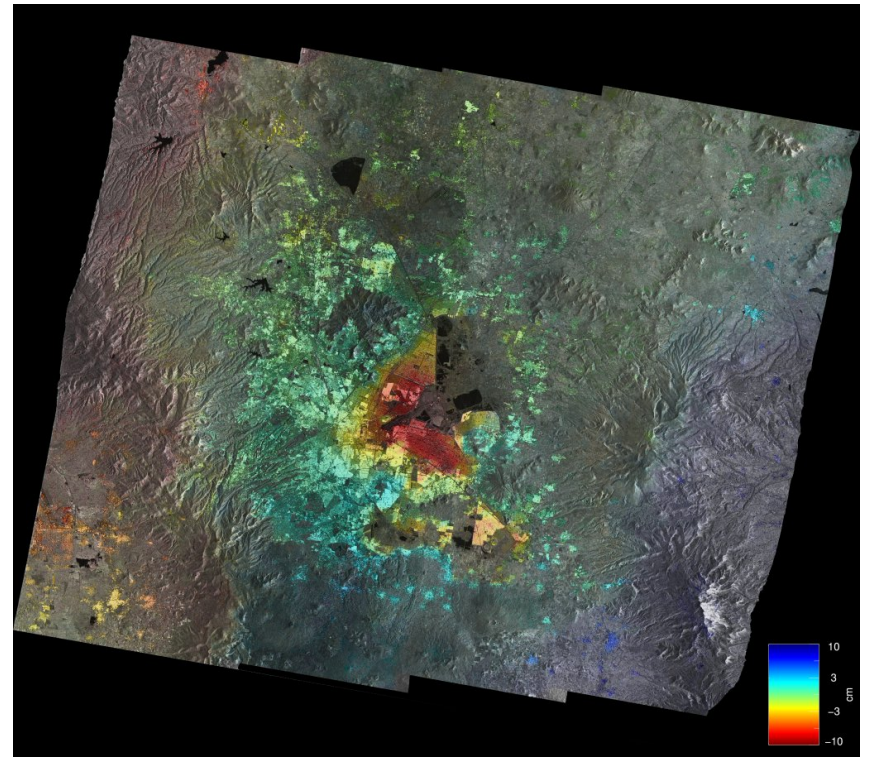

Fig. 6. Estimated subsidence over Mexico City obtained with two TOPS images acquired with 4 months difference. A deformation greater than $10 \mathrm{~cm}$ can be observed in some city areas. The swath is $100 \mathrm{~km}$ ground range.

\subsection{The TOPS Mode}

The TOPS (Terrain Observation by Progressive Scans) mode was first demonstrated in orbit by TSX in 2007. Since then, many acquisitions have been performed, demonstrating the advantages of this wide-swath mode as compared to ScanSAR. TAXI includes the kernel to process TOPS data, which is shared with the sliding spotlight and ScanSAR processors [2]. Fig. 6 shows an example of a differential interferogram over Mexico City, where subsidence due to groundwater extraction on some parts of the city area can be clearly observed.

\subsection{Extended High Resolution Modes}

The use of all the azimuth patterns of TSX allows a variation of $\pm 2.21^{\circ}$ without having to steer the satellite. On the other hand, the operational spotlight products exploit a maximum variation between $\pm 0.75^{\circ}$, which keeps the azimuth ambiguities under control. Therefore, if the larger angle span is exploited, higher resolutions and/or scene extensions are possible. Fig. 7 shows a sliding-spotlight image over Almería, Spain, with an azimuth extension of $10 \mathrm{~km}$ and a nominal azimuth resolution of $0.71 \mathrm{~m}$. Note that the sliding spotlight (SL) operational product has an azimuth extension of $10 \mathrm{~km}$ with $1.7 \mathrm{~m}$ azimuth resolution, while the numbers for the high resolution spotlight (HS) operational product are $5 \mathrm{~km}$ and 1.1 $\mathrm{m}$, respectively.

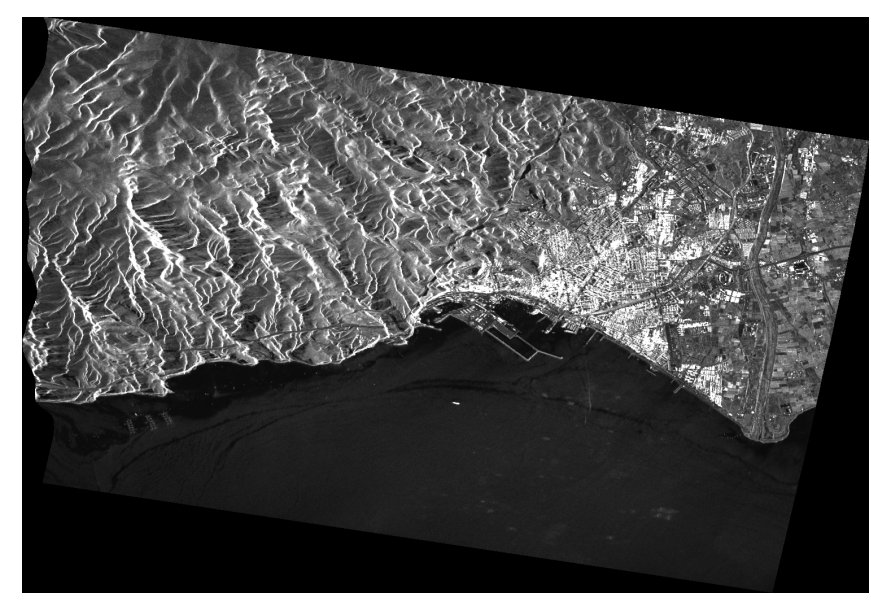

Fig. 7. Geocoded sliding spotlight image over Almería with $0.71 \mathrm{~m}$ nominal azimuth resolution and $10 \mathrm{~km}$ in azimuth.

\section{CONCLUSION}

TAXI is a versatile interferometric processing chain developed at DLR-HR for experimental TanDEM-X product evaluation. It has a special focus on the bistatic processing chain, as well as being able to process the operational products. The flexibility in the implementation of TAXI eases the extension and integration of new modules, hence becoming the perfect platform to process and evaluate new and challenging experiments. This contribution has further addressed several aspects concerning the synchronisation and focussing of bistatic data, while some experiment examples performed by TSX and processed by TAXI have been shown.

\section{REFERENCES}

[1] G. Krieger, A. Moreira, H. Fiedler, I. Hajnsek, M. Werner, M. Younis, and M. Zink, "TanDEM-X: A satellite formation for high-resolution SAR interferometry," IEEE Trans. Geosci. Remote Sensing, vol. 45, no. 11, pp. 3317-3341, Nov. 2007.

[2] P. Prats, R. Scheiber, J. Mittermayer, A. Meta, and A. Moreira, "Processing of sliding spotlight and TOPS SAR data using baseband azimuth scaling," IEEE Trans. Geosci. Remote Sensing, vol. 48, no. 2, pp. 770-780, Feb. 2010.

[3] A. Moreira, J. Mittermayer, and R. Scheiber, "Extended chirp scaling algorithm for air- and spaceborne SAR data processing in stripmap and ScanSAR imaging modes," IEEE Trans. Geosci. Remote Sensing, vol. 34, no. 5, pp. 1123-1136, Sept. 1996.

[4] R. Bamler, F. Meyer, and W. Liebhart, "Processing of bistatic SAR data from quasi-stationary configurations," IEEE Trans. Geosci. Remote Sensing, vol. 45, no. 11, pp. 3350-3358, Nov. 2007.

[5] Y. L. Neo, F. Wong, and I. G. Cumming, "A two-dimensional spectrum for bistatic SAR processing using series reversion," IEEE Geosci. Remote Sensing Lett., vol. 4, no. 1, pp. 93-96, Jan. 2007. 